\title{
EchoGéo
}

$38 \mid 2016$

Varia

\section{Après le boom : la laborieuse mise en œuvre de nouvelles régulations dans le secteur minier guinéen}

Johannes Knierzinger

\section{CpenEdition}

Journals

Electronic version

URL: https://journals.openedition.org/echogeo/14758

DOI: 10.4000/echogeo.14758

ISSN: 1963-1197

Publisher

Pôle de recherche pour l'organisation et la diffusion de l'information géographique (CNRS UMR 8586)

Electronic reference

Johannes Knierzinger, "Après le boom : la laborieuse mise en œuvre de nouvelles régulations dans le secteur minier guinéen", EchoGéo [Online], 38 | 2016, Online since 13 December 2016, connection on 01 August 2021. URL: http://journals.openedition.org/echogeo/14758 ; DOl: https://doi.org/10.4000/ echogeo.14758

This text was automatically generated on 1 August 2021.

EchoGéo est mis à disposition selon les termes de la licence Creative Commons Attribution - Pas d'Utilisation Commerciale - Pas de Modification 4.0 International (CC BY-NC-ND) 


\title{
Après le boom : la laborieuse mise en œuvre de nouvelles régulations dans le secteur minier guinéen
}

\author{
Johannes Knierzinger
}

1 Depuis 2003, après une longue phase de libéralisation des codes miniers qui avait débuté dans les années 1980 et au sein de laquelle on identifie le plus souvent trois "générations» de codes (Campbell, 2004), au moins une douzaine des pays africains ont adopté de nouvelles réglementations minières qui se distinguent visiblement du caractère libéral des codes précédents. Cette nouvelle génération de réglementations concerne la Guinée, le Zimbabwe, l'Afrique du Sud, la Zambie, la Tanzanie, le Congo RDC, le Ghana, l'Angola, le Mozambique et le Burkina Faso'. En réaction au bouleversement mondial des relations entre les pays producteurs de ressources et les pays consommateurs qui avait commencé à la fin des années 1990, ces pays ont fixé des taxes minières plus élevées et intégré des réglementations de local content plus strictes dans leurs cadres juridiques. Beaucoup d'autres pays africains ont introduit l'obligation d'utiliser de la main-d'œuvre locale, renforcé leurs attentes écologiques et sociales et amélioré leur législation concernant le développement local. Dans la plupart des cas, ces changements ont été déclenchés par des mouvements sociaux et des grèves. En même temps, surtout du fait de la concurrence accrue au sein du secteur minier, les grandes entreprises minières ont commencé à adopter des mesures de RSE (responsabilité sociétale des entreprises) qui étaient déjà en pratique dans nombre d'autres secteurs. Cependant, avant que la population ne puisse profiter de cette «fenêtre des possibilités ", plusieurs obstacles restent à franchir :

- Premièrement, la fin du « supercycle » chinois a poussé de nombreuses entreprises minières à réduire leur production, ce qui a conduit à de nombreux rétropédalages législatifs partout en Afrique, que ce soit en termes de taxation, de clauses sociales et environnementales ou encore de participation locale (Open Society Institute of Southern Africa et al., 2009, p. 12; Global Policy Forum Europe, 2011, p. 5). 
- Deuxièmement, une fois un code minier établi, il nécessite encore des textes d'application longs à mettre en place ; de plus, le code doit être intégré aux conventions minières, qui sont souvent protégées contre les changements législatifs par des clauses de stabilité.

- Troisièmement, après ces procédures, les nouvelles lois doivent être réellement appliquées et contrôlées, ce qui n'est souvent pas le cas. À cela s'ajoutent des pratiques comme l'indemnisation, qui ne font pas encore partie intégrante des législations nationales mais qui sont elles aussi devenues de plus en plus importantes et fréquentes avec la hausse des prix des matières premières.

2 L'article retrace ce chemin, depuis les prises de décisions nationales et internationales jusqu'à leur mise en pratique à l'échelle locale. Le cas de la Guinée s'est révélé particulièrement approprié pour illustrer cette problématique car elle possède les plus grandes réserves de bauxite au monde et constitue le cinquième producteur mondial de ce minerai (United States Geological Survey, 2016). Dans la conclusion, l'auteur compare la situation dans ce pays avec d'autres pays africains, aborde les discussions théoriques et évalue des potentiels d'amélioration.

Cette thématique s'inscrit dans les discussions sur l'affaiblissement croissant des États (c'est à dire des gouvernements et des institutions étatiques) avec l'amplification du commerce international ou de ce que l'on appelle communément la mondialisation. L'idée que la mondialisation conduirait à un affaiblissement du pouvoir étatique est devenue si dominante que, telle une prophétie autoréalisatrice, elle conduit déjà ellemême de fait à une réduction des pouvoirs particuliers de l'État, notamment concernant la politique sociale et la redistribution des richesses. C'est exactement cette confusion discursive entre État et politique sociale ou, en d'autres termes, la découverte que l'État peut être fort et antisocial, qui pousse depuis longtemps des théoriciens à mettre en garde contre une tendance à la victimisation de l'État face à un capitalisme considéré comme prédateur (voir par exemple Kannankulam, 2006, sur la critique de Nicos Poulantzas). Dans le contexte africain, cette victimisation de l'État est particulièrement forte et ce n'est que récemment qu'elle a commencé à être remise en question, notamment sur la base des écrits de la politologue Béatrice Hibou (Hibou, 1999a ; Hibou, 1999b ; Hönke, 2009 ; Williams, 2010). Le présent article s'inspire de ces critiques tout en s'interrogeant sur l'applicabilité d'une telle analyse pour le cas de la Guinée.

Dans son analyse des mines en RDC, Jana Hönke (2009, p. 12-16) se base sur les idées de Béatrice Hibou (1999a; 1999b) afin de montrer que l'État de la RDC est effectivement devenu plus puissant pendant la récente phase de dérégulation et de privatisation en utilisant ces dernières comme des instruments d'une "politique de décharge ", terme emprunté à Max Weber. Martin Williams (2010, p. 128) fait un constat similaire dans le cas des mines d'or au Sénégal. Pour la Guinée, ce constat semble trop éloigné de la réalité. Les exemples présentés dans cet article suggèrent plutôt une situation de fragmentation de l'espace politique qui s'amplifie avec la multiplication des décideurs externes.

5 L'analyse se base sur cinq années de recherches menées sur les mines de bauxite en Guinée dans le cadre d'une thèse, dont six mois de recherches (février 2012 et janviermai 2014) sur place dans quatre villes minières (Fria, Sangaredi, Kamsar et Débélé/ Kindia) où l'auteur a vécu avec des familles d'employés des mines et effectué plus de 150 interviews semi-structurées avec les acteurs impliqués. 


\section{Avant et après le boom : les codes miniers guinéens de 2011 et 2013}

6 Des mouvements sociaux et des grèves partout dans le pays en raison de la hausse mondiale des prix des ressources conduisent à un mouvement de renouveau en Guinée à partir de 2007, mais en 2008 le pays connait un putsch de l'armée (initialement soutenu par les syndicats mais rapidement répressif) et des grèves prolongées dans la ville minière de Fria. Ces grèves ont abouti à un lock-out ${ }^{2}$ de la part de l'entreprise Rusal, lequel n'est toujours pas levé et a eu des conséquences graves pour la population de cette ville (Knierzinger, 2016a). Combinés à la chute de la demande mondiale de matières premières, ces développements nationaux ont contribué à un changement de cap du gouvernement d'Alpha Condé. En 2011, le gouvernement Condé, élu en novembre 2010, a adopté un nouveau code minier, qui peut être considéré comme une concrétisation des mouvements sociaux qui ont touché la Guinée depuis 2007. Il est de ce fait comparable à de nombreux codes adoptés en même temps dans d'autres pays à faibles revenus. De la même façon, son amendement en 2013 reflète la fin du boom en lien avec le ralentissement économique mondial qui a suivi la crise financière mondiale de 2007.

7 Comparé avec son prédécesseur de 1995, le code de 2013 a tout de même apporté un grand nombre d'améliorations pour la population riveraine des mines comme pour les Guinéens en général. Les nouveautés concernent, entre autres, un nouveau système de calcul des taxes d'exportation moins facilement contournable par les entreprises minières, le calcul se faisant désormais sur la base des indicateurs internationaux (comme par exemple du London Metal Exchange) et non plus sur la base du chiffre d'affaires (République de Guinée, 2013, \$163); l'ancrage de l'initiative pour la transparence dans les industries extractives (ITIE) dans la loi (\$122); une réduction de la spéculation sur les titres miniers $(\$ 26 \& 34)^{3}$; la nationalisation des infrastructures construites par les entreprises « à l'exception de l'outil de production » après 20 ans de production (\$121); l'obligation d'annoncer les plans sociaux six mois avant la fermeture d'une mine (\$131); l'ouverture d'un fonds de développement local $(\$ 130)^{4}$ et la mise en place d'études d'impact (\$30). Conformément au paragraphe 30 , les entreprises minières ont d'ailleurs effectivement lancé depuis quelques années les premières études d'impact environnemental et social dignes de ce nom ${ }^{5}$.

8 Si l'on compare la situation d'aujourd'hui à celle des années 1970 (c'est-à-dire avant la phase des ajustements structurels), deux phénomènes restent pourtant frappants: premièrement, en 1977, la Compagnie des Bauxites de Guinée (CBG), payait à elle seule presque deux fois plus de taxes (en termes réels) que toutes les entreprises minières réunies en 2016, y compris la CBG elle-même, qui a d'ailleurs fortement augmenté sa production depuis (Knierzinger, 2015, p. 226). Cela ne changera pas avec l'application du code minier de 2013. Deuxièmement, et contrairement à la situation sous Sékou Touré entre 1958 et 1984 (Campbell et Clapp, 1995, p. 431-432) et dans d'autres pays en développement $^{6}$, le code minier guinéen de 2013 incite peu les entreprises à transformer les minerais sur place (voire entre autres \$139). Pour le secteur bauxitique en particulier, les différences entre les codes de 2011 et de 2013 sont considérables. Selon différents calculs, les taxes sur la bauxite guinéenne sont retombées de 11-13 dollars par tonne à 4 dollars (Madsen, 2013) 7 . Du point de vue des revenus étatiques, le code de 2013 s'inscrit ainsi dans une continuité avec les trois générations 
de codes miniers libéraux que l'Afrique a connues à partir des années 1980 (Campbell, 2004).

9 Possédant non seulement des énormes réserves de bauxite mais aussi un fort potentiel en énergie hydraulique, la Guinée serait en théorie tout à fait armée pour produire ellemême de l'aluminium à partir de la bauxite. Cet objectif avait déjà été annoncé par les dirigeants guinéens et les colonisateurs français dès avant l'Indépendance, mais du fait des changements politiques et de la gestion défavorable des risques par les entreprises impliquées pendant les 65 dernières années, il n'a jamais été atteint. Par ailleurs, depuis quelques années, de mauvaises expériences dans d'autres pays africains comme le Ghana et l'Afrique du Sud ont remis en question cet objectif. Les grands barrages posent des problèmes écologiques et sociaux importants (relocalisations, maladies, changement de climat au niveau local, émanations de méthane, etc.) et sont particulièrement difficiles à gérer techniquement dans des zones avec des quantités de pluie très fluctuantes. S'y ajoutent souvent des contrats d'énergie très favorables aux producteurs d'aluminium et qui provoquent de fortes tensions sociales (comme par exemple en Afrique du Sud, où un barrage a récemment fermé à cause des revendications de la population). En Afrique, les barrages ne semblent donc constituer une alternative ni écologique ni économique à la production d'énergie à partir du pétrole ou du charbon ( Knierzinger, 2016b).

De l'Indépendance au boom des années 2000, deux grands producteurs de bauxite, Friguia et la Compagnie des Bauxites de Guinée, étaient responsables de 80 à $90 \%$ des exportations guinéennes et par conséquent de la plupart des revenus publics du pays également (Knierzinger, 2015, p. 28) ${ }^{8}$. Friguia est un consortium qui s'était déjà établi pendant la fin de la période coloniale dans les années 1950 ; la Compagnie des Bauxites de Guinée compte, quant à elle, une mine à Sangaredi ainsi qu'une usine et un port à Kamsar, lequel a commencé à exporter de grandes quantités de bauxite en 1973 (voir figure 1). Une troisième entreprise de production, la Compagnie des Bauxites de Kindia (CBK) à Débélé, près de Kindia, a été construite avec l'aide de l'Union Soviétique et a surtout servi d'instrument de troc avec l'Union Soviétique, comme c'est actuellement le cas avec le "modèle Angola" de la Chine (Edinger et Jansso 2008).

11 Sans le ralentissement économique mondial après la crise des surprimes, les Guinéens auraient pu profiter de l'importante augmentation des taxes minières prévue par le code de 2011 et même pousser les entreprises à réaliser une promesse faite dès les années 1950, à savoir le lancement d'une production intégrée de l'aluminium. Ceci ne s'étant pas produit, on peut considérer que la «fenêtre des possibilités" des années 2000 a été trop courte pour sortir du régime extractiviste libéral qui profite avant tout aux acteurs externes. 
Illustration 1 - La production de bauxite en Guinée

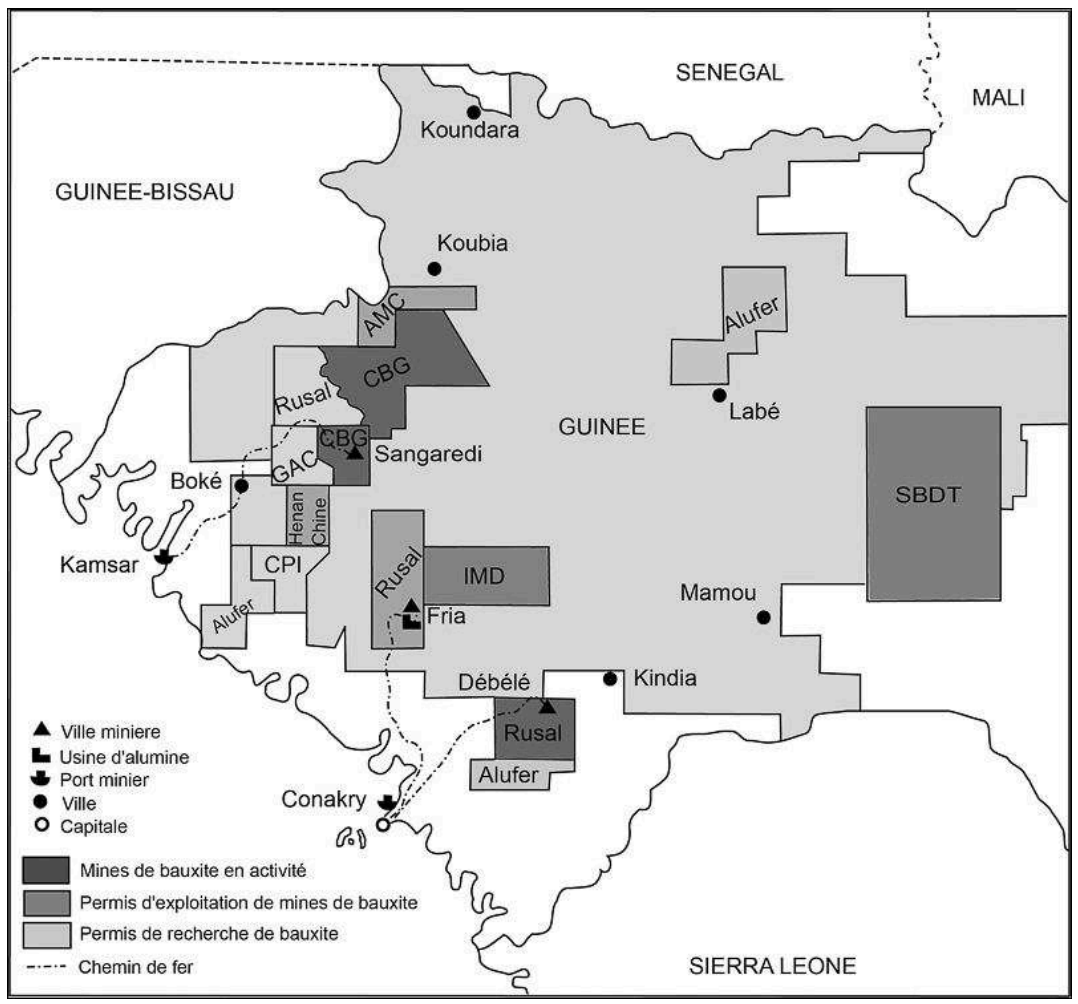

\section{L'application du code}

De nombreuses nouveautés du code de 2013 pourraient certes améliorer la situation de la population sur place mais elles sont soumises aux mêmes changements de relations de pouvoir à l'échelle globale. Plusieurs parties du code doivent d'abord être complétées par des décrets (ou textes) d'application et les conventions entre l'État et les entreprises minières doivent elles aussi être adaptées. En raison de ces obstacles, le code de 2013 pourrait dès lors finir comme son prédécesseur de 1995, c'est à dire fortement déconnecté des pratiques sur place.

En mars 2014, un expert guinéen constatait qu'il manquait encore 39 de ces textes en lien avec le nouveau code minier. Seuls cinq avaient déjà été rédigés, et aucun n'était encore adopté (interview à Kindia, le 13.03.2014; Knierzinger, 2015, p. 136). Du fait de la lenteur des procédures, les textes d'application risquent toujours de se heurter au même obstacle que le code minier, à savoir les changements du contexte politique et économique (inter)national. Avec la baisse tendancielle du prix de l'aluminium depuis 2008 (hormis une courte phase de rebondissement en 2009 et 2010 ; United States Geological Survey, 2016) qui découle de l'énorme surcapacité des usines mondiales ${ }^{9}$, ces textes pourraient devenir des éléments de discorde et n'être dès lors jamais appliqués.

Au-delà des textes d'application, l'ajout de clauses supplémentaires dans les conventions minières préexistantes dépend non seulement du contexte politique et économique mondial, mais également de l'existence éventuelle de "clauses de stabilité" dans les conventions (lesquelles permettent aux entreprises de ne pas appliquer certains des amendements du nouveau code minier) - et jusqu'à un certain point aussi de l'âge des sites de production ${ }^{10}$. 
15 À cela s'ajoute enfin, comme le souligne le géographe Géraud Magrin, une stratégie couramment utilisée par les entreprises minières, qui consiste à annoncer la fermeture prochaine de la mine afin de décourager l'État de se lancer dans le processus administratif complexe que représenterait l'amendement de la convention minière préexistante $^{11}$. En Guinée, c'est ce qui semble s'être produit dans la mine de CBK Rusal à Débélé près de la région de Kindia. Cette mine est connue pour être la mine industrielle avec les standards les plus bas et elle n'a d'ailleurs pas encore connu d'améliorations avec le nouveau code minier. En avril 2014, les travailleurs ont appris que la mine fermerait dans les cinq à dix années à venir et qu'il était dès lors peu probable que la convention minière soit encore amendée d'ici là (Knierzinger, 2015, p. 207).

Pour ces trois raisons, il est probable que le nouveau code reste fortement déconnecté des conventions avec les entreprises et donc des pratiques sur place. C'est d'ailleurs ce qui se passait déjà avec le code minier de 1995. Un rapport de la GIZ, l'agence allemande pour le développement, dénonce une "cacophonie dans la gestion des ressources locales provenant de l'exploitation minière et des carrières " (Diallo et al., 2011, p. 56), " cacophonie " liée à l'existence de textes d'application contradictoires du code minier guinéen de 1995, un code typiquement libéral qui est resté en vigueur jusqu'à 2011 (et dans la pratique jusqu'à la remise de cet article en octobre 2016). D'après cette étude, «dans chaque région ou préfecture, l'autorité administrative applique les textes qui correspondent à ses intérêts particuliers » (ibid.). Dans ce contexte, les collectivités locales n'arrivent à faire valoir leurs droits que lorsque les présidents des communautés rurales de développement (CRD) ou les maires maitrisent les textes (ce qui suppose déjà qu'ils sachent lire, ce qui n'est pas toujours le cas) et disposent d'une forte influence politique (Diallo et al., 2011, p. 56).

\section{La responsabilité sociale des entreprises (RSE) remplace les taxes locales}

Ainsi, sur la question du développement local notamment, ce sont finalement les entreprises qui établissent leurs propres règles : la CBG (Sangaredi et Kamsar) a par exemple fixé sa contribution à 500000 USD par an; de son côté, la Guinea Alumina Corporation (GAC), une compagnie junior d'abord américaine puis rachetée par des investisseurs arabes et qui s'est établie à Sangaredi, investit 500000 à 1000000 USD par an (dans les deux cas ces sommes sont gérées par les sociétés elles-mêmes) et la CBK (Débélé/Kindia) a accepté de payer 100000 USD par an à partir de 2001. Dans tous ces cas, il est très difficile de savoir si ces payements ont effectivement eu lieu car la distinction entre ce qui relève des taxes locales et ce qui relève des mesures de responsabilité sociale des entreprises (RSE) est très floue. Rusal, qui a officiellement repris la mine de Débélé en 2001 ne semble pas avoir payé de taxes locales ni déclaré de mesures RSE jusqu'à la prise du pouvoir d'Alpha Condé en 2010. Après ce tournant politique, elle a pris des mesures allant dans les deux sens (Knierzinger 2015, p. 206).

La CBG paye très peu des taxes locales et décide en même temps comment la commune doit les utiliser. Dans le code minier de 2013 qui n'est pas encore appliqué, la contribution au développement de la communauté locale est fixée à $0,5 \%$ du chiffre d'affaire pour la bauxite et le fer et à $1 \%$ pour l'or et les diamants (République de Guinée, 2013, \$130). Dans certaines villes minières guinéennes comme Siguiri, Dinguiraye (les deux possèdent des mines industrielles d'or) et Kindia (Rusal), ces taxes 
parviennent déjà aux collectivités, même si la nouvelle loi ne précise pas si cette " contribution » doit être payée en investissement direct ou versée sous forme de taxes locales (Knierzinger, 2016, p. 236).

19 Afin de clarifier la relation entre les investissements "publics » et " privés » dans les villes minières, l'auteur a examiné les budgets de 2011-2013 de la commune urbaine de Kamsar, qui s'est construite autour d'une usine de transformation de la bauxite de la CBG venant de Sangaredi. Cette étude a montré que près de $99 \%$ des infrastructures d'usage public dans cette ville de plus de 100000 habitants avaient été construites et entretenues par des entreprises étrangères. Ce pouvoir infrastructurel privé rend les élections locales presque absurdes et représente un moyen de pression important, par exemple dans les négociations avec les syndicats (Knierzinger, 2015, p. 237-241).

Ce remplacement des taxes locales par des initiatives privées volontaires crée donc un obstacle supplémentaire entre le nouveau code minier et sa mise en pratique.

\section{Suivi des pratiques des entreprises minières : manque de moyens et manque d'information}

21 En raison de ces insuffisances dans la conception et dans l'application du code, peu de pratiques sur place résultent du nouveau code minier. Quant aux pratiques encadrées par la loi, le problème de leur contrôle ensuite se pose. À l'heure actuelle, il n'existe guère de suivi étatique des pratiques des entreprises minières en Guinée. Cela concerne tant la relocalisation des populations autour des mines que les problèmes de santé liés à l'exploitation, la pollution et la destruction des champs et des cours d'eau, les accidents de circulation liés à l'exploitation ou encore la déforestation. La société GAC, qui n'a pas encore commencé à exploiter la bauxite, a, par exemple, relocalisé un grand nombre de paysans dans la sous-préfecture de Sangaredi et créé à cet effet un Comité de suivi environnemental et de la réinstallation qui regroupe des représentants de l'entreprise, des administrateurs et des membres du conseil municipal. Mais une représentante de GAC interviewée à ce sujet critiquait vivement le fait que ces mécanismes de contrôle tournent en réalité à l'absurde dans la pratique car les entreprises minières semblent forcées de soudoyer les contrôleurs étatiques - pas forcément pour que ceux-ci rédigent des rapports favorables mais déjà simplement pour qu'ils acceptent de se déplacer (Knierzinger, 2015, p. 269).

Ce manque de contrôle, qui relève tout d'abord d'un manque de moyens (manque de véhicules, coûts des transports) mais aussi d'un manque d'information, se retrouve dans plusieurs autres secteurs des villes minières de Guinée. Au cours de ses recherches à Kindia/Débélé, l'auteur a pu s'entretenir avec les principaux acteurs locaux de la mine, de l'administration locale et régionale, de syndicats et avec d'autres acteurs de la société civile. Aucun d'entre eux n'avait connaissance de la convention minière de Rusal CBK, alors que celle-ci est pourtant publiée sur internet depuis février 2013 (plus d'un an avant l'enquête de l'auteur) par le Comité Technique de Revue des Titres et Conventions Miniers. De plus, aucun des acteurs interrogés ne pouvait (ou ne voulait) expliquer sur quelles bases Rusal CBK paye ses taxes locales ou comment elle procède au reboisement dans la région. Cette absence de communication n'a cependant rien de nouveau et a déjà provoqué plusieurs conflits violents au cours des dix dernières années. 


\section{La situation dans les villages et les pratiques d'indemnisation locale} la destruction de leurs biens. Pour cela, il n'existe pas de régulations nationales et les entreprises se basent soit sur leurs propres grilles de mesures, soit (de plus en plus) sur les propositions de la Banque Mondiale et du FMI. La situation des paysans vivant autour des mines est souvent déplorable. Le problème le plus grave (mais pas le seul) concerne l'approvisionnement en eau. Dans nombre de villages, les forages initialement construits par les entreprises pour pallier la modification des cours d'eau due au dynamitage n'ont pas été entretenus et la population se voit donc forcée de parcourir de longues distances pour aller chercher de l'eau. Le dynamitage provoque également des fissures dans les maisons et fait fuir le gibier. La poussière omniprésente dans l'air autour des mines provoque des problèmes respiratoires, des infections des yeux et fait baisser fortement la productivité de l'agriculture. À cela s'ajoute un problème d'accessibilité de ces villages dû à la progression des mines. À Sangaredi et à Kamsar, malgré l'accord de collaboration déjà en vigueur entre l'Université de Leipzig et la Compagnie des Bauxites de Guinée et en dépit de plusieurs demandes et relances de la part de l'auteur, il n'a pas été possible d'avoir accès à la moindre étude d'impact. Ces études sont pourtant censées être menées avant la construction de nouvelles mines ou leur extension. Du fait du manque d'information, les nombreuses relocalisations dans la sous-préfecture de Sangaredi ont souvent des conséquences graves pour les populations concernées (Knierzinger, 2015, p. 255-270).

Dans la plupart des cas, les paysans affectés par ces problèmes n'arrivent pas à faire valoir leurs droits, et ce pour différentes raisons : bien souvent, ils ne les connaissent pas, car les schémas d'indemnisation ne sont pas publiés. Par ailleurs les entreprises n'indemnisent la destruction de biens privés que si la victime dépose une plainte écrite à la sous-préfecture concernée. Cela représente un obstacle considérable pour des paysans majoritairement analphabètes. Enfin, les paysans des zones rurales concernées n'ont souvent ni les compétences, ni les moyens matériels nécessaires pour faire entendre leur voix. Des problèmes aussi élémentaires que les transports ou l'accès au carburant rendent par exemple tout déplacement en ville quasiment impossible. Au regard de tous ces obstacles, on comprend mieux pourquoi les plaintes sont si rares. Un technicien interrogé au Service environnement de la CBG ne connaissait par exemple qu'un seul cas d'indemnisation à Sangaredi en 2012 et 2013. Le responsable des Projets communautaires à Kamsar a, pour sa part, enregistré en tout et pour tout 18 plaintes dans l'ensemble des collectivités où la CBG opère depuis 2010 (Knierzinger, 2015, p. 257).

À Sangaredi l'auteur a eu l'occasion de suivre de près un cas de contestation après la destruction d'une plantation de 40 anacardiers (arbres à noix de cajou) par des agents de la CBG. L'administrateur de la sous-préfecture concernée possédait une liste d'indemnisations correspondant aux différents types de plantations qui datait de 1987 et prévoyait une indemnisation de $3000 \mathrm{GNF}$ ou 0,33 USD par arbre. En prenant en compte une inflation de $5 \%$ par année sans prêter attention à l'effet d'intérêt composé, l'administrateur chargé des indemnisations en arrivait à un prix actuel de 7000 GNF (ou environ 1 USD) par arbre. 

compte le taux d'inflation exact et effectué un calcul correct, le paysan aurait eu droit à 60000 GNF par arbre, soit 343 USD et non 40 USD pour les 40 arbres. Pendant les «négociations » entre l'administrateur, le paysan et l'auteur, le premier a également montré les grilles d'indemnisation de la GAC qui s'orientent sur les recommandations de la Banque Mondiale. Dans le périmètre du permis d'exploitation de la GAC, le même paysan aurait reçu une indemnisation de 460 USD (Knierzinger 2015, p.258). Ces chiffres montrent bien l'arbitraire et le manque de transparence des schémas d'indemnisation existants. Le paysan concerné ne savait ni calculer l'intérêt composé, ni parler suffisamment français pour suivre les détails de la négociation. C'est ainsi qu'une procédure censée aboutir à de simples payements selon des critères compréhensibles et publics se transforme en négociation opaque laissant beaucoup de marge de manœuvre à la corruption au niveau local.

Dans notre exemple, le cultivateur expliquait qu'il avait d'abord eu affaire à des «collègues» de l'administrateur responsable et à des employés de la CBG qui lui avaient proposé $5000 \mathrm{GNF}$ par arbre, soit moins de 30 USD pour l'ensemble de la plantation détruite. Lorsqu'il a menacé de bloquer la route, les mêmes personnes ont doublé leur proposition. Après un deuxième échec des négociations, il s'est vu proposer un «petit contrat» de travail de deux mois par la CBG. Le cultivateur refusa de nouveau et, à ce point des négociations, avait déjà tout son village derrière lui, prêt à bloquer les travaux. Face à ces menaces, l'administrateur a finalement annoncé au cultivateur qu'il n'avait droit à aucune compensation, arguant du fait qu'il aurait dû enregistrer au préalable son terrain, au prix de $150000 \mathrm{GNF}$, et qu'il aurait également dû payer 200000 GNF supplémentaires pour une analyse des dégâts, par ailleurs nonremboursables en cas de constat négatif. Cela faisait déjà 50 USD, soit presque le double de la première proposition. Et comme si cela ne suffisait pas, l'administrateur annonça également que l'enregistrement de la plantation d'anacardiers n'était de toute façon pas possible parce que le paysan ne possédait que deux hectares de culture au lieu des cinq nécessaires pour pouvoir faire accréditer une plantation.

Le cultivateur ne possédait donc finalement une base de droit ni «dur " (les indemnisations étant seulement réglées au niveau des entreprises) ni « souple » pour ses demandes. Il pouvait dès lors soit faire le choix de croire en la bonne foi des négociateurs, soit continuer à bloquer les travaux. On ne connaît malheureusement pas la fin de cette histoire, mais il est très probable que les "négociateurs" aient finalement trouvé une solution «acceptable». Pendant ses recherches, l'auteur a rencontré un grand nombre de cas semblables qui avaient tous en commun que seule une partie des acteurs de la négociation connaissait les règles du jeu (ou prétendait les connaître) et avait suffisamment de connaissances (parler, écrire, calculer, etc.) pour y jouer.

\section{Conclusion}

29 L'apparition simultanée de la nouvelle vague des codes miniers et la montée de la «responsabilité sociale des entreprises» dans le secteur minier à partir des années 2000 n'est ni un hasard, ni l'expression d'un nouvel angélisme soudain du côté des investisseurs. Comme les autres conséquences de la nouvelle course aux matières premières (militarisation, politique agressive des accords de libre-échange, 
investissements accrus dans le recyclage), ce double phénomène illustre l'évolution des rapports de force à l'échelle mondiale, entre États producteurs et acheteurs, patrons et travailleurs, gouvernements et populations etc. En Guinée, comme dans de nombreux autres pays, ces développements ont suscité de nouveaux espoirs au sein de la population qui a cru qu'elle pourrait réellement tirer profit des richesses de ses soussols. Pourtant, les cas présentés dans cet article montrent à quel point la mise en application de ces innovations a été complexe et lente depuis l'ouverture de cette «fenêtre des possibilités». Le fait que l'ouverture de cette fenêtre ait été particulièrement courte a réduit la chance de voir ce code se traduire dans des textes d'application et dans les conventions minières établies entre l'État et les entreprises. Les pratiques de contrôle et d'indemnisation montrent quant à elles qu'une « véritable » application locale du code nécessiterait encore beaucoup plus de temps et de ressources. Ces constats suggèrent que même dans une phase de hausse des prix des matières premières, les effets du paradoxe de l'abondance semblent peser trop lourd dans un pays doté d'institutions politiques aussi faibles. Cette notion de faiblesse nous ramène aux réflexions théoriques esquissées dans l'introduction de cet article.

Dans son analyse des activités minières dans la RDC, Jana Hönke (2009) critique la simple victimisation de l'État en constatant que la dérégulation et la privatisation viennent, au moins en partie, d'une "politique de décharge " menée par les élites étatiques elles-mêmes (Hibou 1999a, 1999b ; Williams, 2010) et que l'État de la RDC est effectivement devenu plus puissant pendant la récente phase de dérégulation. Les exemples présentés concernant la Guinée suggèrent des relations de pouvoir assez différentes. Même après la montée des nouveaux investisseurs des pays BRICS qui auraient $\mathrm{pu}$ théoriquement agrandir la marge de manœuvre des responsables politiques, le gouvernement guinéen ne peut guère être comparé à un marionnettiste qui tirerait les ficelles dans l'ombre de la scène. Si l'on tient à cette métaphore, il ressemblerait plutôt à une marionnette qui semble s'être emmêlée dans les ficelles des nombreux marionnettistes la manipulant selon des intérêts divergents. En ce sens, l'idée développée par Michael Shafer d'un "État entonnoir»- dans lequel les «dirigeants doivent adopter des tactiques de survie qui limitent leur autonomie, détournent les ressources initialement destinées au développement et à la diversification et réduisent leur capacité de mener les transformations sociales nécessaires » (Shafer, 1986, p. 946-947, traduction : Knierzinger) semble plus adaptée à la situation guinéenne.

31 Au niveau local, la nouvelle vague de codes miniers africains pose également la question des effets secondaires non planifiés de l'application de ces règles. L'exemple des quatre villes minières présentées dans cet article souligne à cet égard à la fois la nécessité d'une harmonisation des régulations locales (notamment en termes d'indemnisations et de taxes) et amène à s'interroger sur la faisabilité d'une telle harmonisation. Une façon de réduire le fossé entre la loi et la pratique pourrait consister en un recours accru aux Agents de Développement Communautaire. Ces agents sont jusqu'à maintenant avant tout chargés d'écrire les budgets et les programmes de développement local, mais ils pourraient également servir à promouvoir et renforcer la transparence et l'équilibre des négociations au niveau national et local.

Sans un véritable renforcement des capacités de contrôle et de pouvoir des administrateurs et élus locaux, il est très probable que la tendance actuelle consistant à 
verser une partie importante des taxes directement aux collectivités locales soit à nouveau remise en question. Etant responsables surtout envers leurs investisseurs et leurs acheteurs, les entreprises minières risquent fort de faire pression pour le remplacement de leurs taxes par les mesures RSE si les administrateurs locaux ne peuvent leur garantir que ces taxes parviennent bel et bien à la population. Au lieu de payer leurs taxes, les entreprises multinationales pourraient ainsi remplacer encore davantage les institutions publiques qu'elles ne le font déjà - même contre le gré de leurs dirigeants. Contrairement à d'autres pays (comme par exemple au Burkina Faso), un tel recul démocratique serait aisément envisageable en Guinée car le code minier ne prescrit pas clairement la forme (en taxes locales ou sous la forme de mesures de RSE) qu'est censée prendre la contribution au développement local.

\section{BIBLIOGRAPHY}

Agence Ecofin, 2013. Guinée: Alpha Condé annule 818 permis miniers pour assainir et relancer le secteur extractif. En ligne : http://www.agenceecofin.com/gestion-publique/2012-8136-guinee-alphaconde-annule-818-permis-miniers-pour-assainir-et-relancer-le-secteur-extractif , consulté le 3 décembre 2013.

BBC News, 2016. Zimbabwe clamps down with its indigenisation laws. BBC News, 1 avril 2016. En ligne : http://www.bbc.com/news/business-35942027, consulté le 10 avril 2016.

Campbell B.-K., 1986. Bauxite Bargaining. The consequences for a bauxite-producing country of the renegotiation of the bauxite levy: The example of Guinea. Vienna, United Nations Industrial Development Organization.

Campbell B.-K. (ed.), 2004. Regulating mining in Africa: For whose benefit? Discussion Paper 26, Uppsala, Nordic Africa Institute.

Campbell B.-K., 2009. Guinea and Bauxite-Aluminium: the Challenges of Development and Poverty. In Campbell B.-K., Mining in Africa. Regulation \& Development. Ottawa, International Development Research Centre, p. 66-119.

Campbell B.-K., Clapp J., 1995. Guinea's Economic Performance under Structural Adjustment: Importance of Mining and Agriculture. The Journal of Modern African Studies, vol. 33, n 3, p. $425-449$.

Central Intelligence Agency, 2015. The World Factbook. En ligne : https://www.cia.gov/library/ publications/the-world-factbook/, consulté le 28 mai 2015.

Diallo B., 2010. Crise à Fria : l'ancien Directeur de l'usine El hadj Lancei Traoré se prononce ! En ligne : http://www.africaguinee.com/articles/2014/06/02/crise-fria-l-ancien-directeur-de-lusine-el-hadj-lancei-traore-se-prononce, consulté le 28 janvier 2015.

Diallo M.-C. et a.l, 2011. Les enjeux de la gouvernance du secteur minier en Guinée. Conakry, Coopération Guinée Allemagne.

Economic Commission for Africa; African Union, 2013. Making the Most of Africa's Commodities: Industrializing for Growth, Jobs and Economic Transformation. Economic report on Africa 2013. Addis 
Ababa, ECA. En ligne : http://www.uneca.org/sites/default/files/publications/ unera_report_eng_final_web.pdf, consulté le 24 janvier 2015.

Edinger H., Jansso J., 2008. China's 'Angola Model' comes to the DRC. The China Monitor, vol. 34, p. 4-7.

Fuchs P., 2012. Die Rohstoffpolitik Deutschlands und der EU. Nachschub für die imperiale Lebensweise. Politische Ökologie, vol. 129, p. 43-48.

Global Policy Forum Europe, 2011. Anforderungen an eine zukunftsfähige Rohstoffstrategie. Stellungnahme zivilgesellschaftlicher Organisationen zur Rohstoffstrategie der Bundesregierung. Bonn. En ligne : http://www.bicc.de/fileadmin/Dateien/pdf/press/2010/

Anforderungen_an_eine_zukunftsfaehige_Rohstoffstrategie.pdf

Guinéeinformation, 2014. Interview du professeur Alpha Condé à Genève, sur Rio Tinto, Vale, Simandou, Rusal, Tony Blair, BSGR de Beny Steinmetz, Georges Soros... En ligne : http://guineeinformation.fr/ index.php/guinee/item/753-interview-du-professeur-alpha-conde-a-geneve-sur-rio-tinto-valesimandou-rusal-tony-blair-bsgr-de-beny-steinmetz/753-interview-du-professeur-alpha-conde-ageneve-sur-rio-tinto-vale-simandou-rusal-tony-blair-bsgr-de-beny-steinmetz, consulté le 14 août 2014.

Hibou B., 1999a. La décharge, nouvel interventionnisme ? Politique Africaine, vol. 73, p. 6-15.

Hibou B., 1999b. La Privatisation des États. Paris, Karthala.

Hönke J., 2009. Transnational pockets of territoriality. Governing the security of extraction in Katanga (DRC). Working Paper Series of the Graduate Centre Humanities No.2, Leipzig, Leipziger Universitätsverlag.

Husband C., McMahon G., van der Veen P., 2009. The Aluminum Industry in West and Central Africa. Lessons Learned and Prospects for the Future. Extractive Industries and Development Series \#13, Washington, D.C, World Bank.

Kannankulam J., 2006. Autoritärer Etatismus im Neoliberalismus. Zur Staatstheorie von Nicos Poulantzas. Hamburg, VSA.

Kappès-Grangé A., Soudan F., 2012. Alpha Condé : "J'ai hérité d'un pays sans État". Jeuneafrique.com, 16 juillet 2012. En ligne : http://www.jeuneafrique.com/Article/ JA2687p024-030.xmlo/, consulté le 9 août 2014.

Kleijn E.-G.-M., 2012. Materials and energy: a story of linkages. Leiden, thèse de doctorat.

Knierzinger J., 2015. Corporate control in Guinean bauxite towns: How to jump off a lion. Leipzig/Paris, thèse de doctorat.

Knierzinger J., 2016a. Fria in Guinea: A Dismissed Bauxite Town. Stichproben, vol. 30/2016, p. 137-161. En ligne : http://stichproben.univie.ac.at/aktuelle-ausgabe/, consulté le 23 août 2016.

Knierzinger J., 2016b. Bauxit und Aluminium aus Afrika. Ausbeutung auf Umwegen. In Fischer K, Jäger J., Schmidt L., Rohstoffe und Entwicklung. Aktuelle Auseinandersetzungen im historischen Kontext. Vienne, new academic press, p. 203-220.

Luning S., 2012. Processing Promises of Gold: A Minefield of Company-Community Relations in Burkina Faso. Africa Today, vol. 58, n 3, p. 22-39.

Madsen M., 2013. Guinea cuts taxes, royalties in revised mining code in bid to woo investors. Steelfirst.com, 11 avril 2013. En ligne : http://www.steelfirst.com/Article/3189167/Industry-andCompanies/Guinea-cuts-taxes-royalties-in-revised-mining-code-in-bid-to.html, consulté le 27 janvier 2015. 
Magrin G., 2013. Voyage en Afrique rentière. Paris, Publications de la Sorbonne.

Natural Resource Governance Institute, 2013. Financials. En ligne : http://

www.revenuewatch.org/about/financials, consulté le 6 octobre 2013.

Prichard W., 2009. The Mining Boom in Sub-Sharan Africa. Continuity, Change and Policy Implications. In Southall R., Melber H., A New Scramble for Africa? Imperialism, Investment and Development. Scottsville, Kwazulu-Natal, University of KwaZulu-Natal Press, p. 240-273.

Open Society Institute of Southern Africa et al., 2009. Breaking the Curse: How Transparent Taxation and Fair Taxes Can Turn Africa's Mineral Wealth into Development. Johannesburg. En ligne : http:// www.christianaid.org.uk/Images/breaking-the-curse.pdf

République de Guinée, 2006. Annexe C de la convention du 03 novembre entre la République de Guinée et "Rousski Alumini" pour la réhabilitation, l'extension et l'exploitation des gisements de bauxite de Kindia. Conakry.

République de Guinée, 2013. Code Minier 2011 amendé. Conakry.

Shafer D.-M., 1986. Undermined: The Implications of Mineral Export Dependence for State Formation in Africa. Third World Quarterly, vol. 8, n 3, p. 916-952.

Shearman and Sterling, 2014. Mozambique's New Mining Law. En ligne : http:// www.shearman.com/ /media/Files/NewsInsights/Publications/2014/10/Mozambiques-NewMining-Law--A-ReBalancing-Act-PDF-102714.pdf, consulté le 10 avril 2016.

Speight R., Varax M., Dorin A., 2012. Recent Reforms of the Mining Code and Model Mining Convention in Mauritania. Legal Update May 2012. Mayer Brown. En ligne : http://www.mayerbrown.com/files/ Publication/01248033-d710-406d-a4b0-2fc531e0185d/Presentation/PublicationAttachment/ c67ee091-35dd-4a09-9bf3-bd2959c42791/mauritania-mining-reform-May12.pdf, consulté le 10 avril 2016.

The Moscow Times, 2015. Ukraine Supreme Court Upholds Nationalization of RusAl Aluminum Plant. The Moscow Times, 12 mars 2015. En ligne : http://www.themoscowtimes.com/business/ article/ukraine-supreme-court-upholds-nationalization-of-rusal-aluminum-plant/517382.html, consulté le 10 avril 2016.

United States Geological Survey, 2016. En ligne : http://www.usgs.gov/, consulté le 22 août 2016.

Williams M. 2010. The gold standard of governance: mining, decentralization, and State power in Senegal. Politique Africaine, $\mathrm{n}^{\circ} 117$, p. 127-148.

WTO, 2013. Dispute Settlement: Dispute DS394 China - Measures Related to the Exportation of Various Raw Materials. En ligne : http://www.wto.org/english/tratop_e/dispu_e/cases_e/ds394_e.htm, consulté le 16 septembre 2013.

\section{NOTES}

1. Pour l'Afrique du Sud et la RDC, voir Prichard, 2009, p. 268 ; pour l'Angola, voir Economic Commission for Africa et African Union, 2013, p. 99; pour la Mauritanie, voir Speight, Varax et Dorin, 2012; pour le Mozambique, voir Shearman and Sterling, 2014 ; et pour les développements récents au Zimbabwe, voir BBC News, 2016. Pour les autres pays, voir Open Society Institute of Southern Africa et al., 2009 ; et un article de Sarah Katz-Lavigne intitulé "The renegotiation window: Resource contract renegotiations in the mining industry in Africa from 2000 to the present", à paraitre dans Resources Policy en 2016. 
2. Ce lock-out s'est traduit par le verrouillage de l'usine, l'arrêt de la production et la cessation du paiement des salaires et autres indemnités, contre l'avis des travailleurs.

3. En vertu de ce paragraphe, les compagnies sont forcées de lancer réellement l'exploration six mois et l'exploitation quatre années maximum après l'acquisition des permis et la signature des contrats (Guinée information, 2014).

4. En vertu de ce paragraphe, les usines sont tenues de verser 0,5 à $1 \%$ de leur chiffre d'affaires pour la «communauté locale ». Le terme "communauté » a été récemment critiqué pour son imprécision qui donne la possibilité de faire jouer la concurrence entre les groupes concernés par l'exploitation (Magrin? 2013, p. 199 ; Luning, 2012, p. 26). En Guinée, le terme de communauté locale ne définit pas une structure officielle de la décentralisation et ne doit pas être confondu avec les communautés rurales de développement (CRD) qui s'étendent sur le même territoire que les sous-préfectures.

5. Pourtant, étant donné que les agences sont payées par les entreprises elles-mêmes, le risque subsiste que les études critiques ne soient finalement pas prises en compte dans les rapports finaux.

6. Le cas de l'Indonésie est un exemple particulièrement parlant: en juin 2012, suite à une politique de réduction des exportations menée par l'Indonésie, la part des importations de bauxite indonésienne en Chine est passée de $80 \%$ de toutes les importations à presque zéro en moins d'un mois. Or, ces restrictions d'exportations indonésiennes correspondaient à la même stratégie que celle appliquée au même moment par la Chine à l'Europe dans le cas des terres rares. La presse guinéenne rapporte qu'à partir de ce moment-là, la Chine a essayé encore plus énergiquement qu'auparavant d'avoir accès aux gisements guinéens (Knierzinger, 2015, p. 107).

7. Malgré ces reculades gouvernementales et la pression des entreprises multinationales, les syndicats et autres mouvements sociaux ont également utilisé cette phase de révision pour revendiquer (avec succès) des améliorations concernant l'emploi et le développement local (Knierzinger, 2015, p. 134).

8. Depuis, deux producteurs d'or se sont ajoutés.

9. Les investissements dans le secteur de l'aluminium se font sur le long terme, soit sur une période allant de 10 à 15 ans, et produisent ainsi des « cycles de porc» (Kleijn, 2012, p. 31) énormes, surtout dans des marchés de plus en plus dépendants de la valeur actionnariale à court terme.

10. Le paragraphe $217 \mathrm{du}$ nouveau code minier guinéen différencie trois «ordres" d'amendements. Les premiers doivent être appliqués immédiatement et incluent entres autres la protection de l'environnement et les relations avec les communautés locales. Les amendements de deuxième ordre doivent être appliqués «sur une période de durée négociée mais ne pouvant excéder huit ans » et concernent la formation, l'emploi et la préférence donnée aux entreprises guinéennes. Le troisième ordre concerne entre autre le régime fiscal et n'a pas de date butoir. Comme évoqué précédemment, les « clauses de stabilité » peuvent exclure des changements de premier et deuxième ordre.

11. Cf. Intervention de G. Magrin dans le cadre du workshop "Activités minières en Afrique subsaharienne: quels impacts sociaux et quelles compensations? Enjeux, perspectives et controverses" qui s'est tenu à Ouagadougou du 23 au 25 mars 2016. 


\section{ABSTRACTS}

Since 2003, after a long period of liberalization, more than a dozen African countries have adopted new mining codes which distinguish themselves markedly from their liberal predecessors. Drawing on fieldwork in Guinea, the article retraces the different stages of the implementation process of such a "progressive" mining code, including its modification in 2013 due to the pressure of the mining industry, the adoption of application texts and the adaption of mining contracts, and the introduction of new practises and their supervision in and around the mines.

Depuis 2003, après une longue phase de libéralisation dans le secteur minier, au moins une douzaine de pays africains ont adopté de nouveaux codes miniers. Cette nouvelle " génération " de codes se distingue visiblement du caractère libéral des précédents. À partir de l'exemple de la Guinée avant et après le boom minier des années 2000, l'article retrace les étapes de la mise en œuvre d'un tel code minier depuis son adoption en 2011 jusqu'aux nouvelles pratiques et leur suivi sur place, en passant par la modification du code minier en 2013 et par l'adoption des textes d'application et l'adaptation des conventions minières existantes.

\section{INDEX}

Mots-clés: Guinée, bauxite, code minier, indemnisation

Keywords: Guinea, mining code, compensation, bauxite

\section{AUTHOR}

\section{JOHANNES KNIERZINGER}

Johannes Knierzinger, johannes.knierzinger@univie.ac.at, est chercheur postdoctoral à l'Institut de recherche pour le développement (IRD) et membre de l'UMR Prodig. Il a publié récemment: - Knierzinger J., 2014. The socio-political implications of bauxite mining in Guinea: A commodity chain perspective. The Extractive Industries and Society, 1 (1), p. 20-27.

- Knierzinger J., 2016. Fria in Guinea: A dismissed bauxite town. Stichproben, Vienna Journal of African Studies, Vol. 16, n 30, p. 137-160.

- Knierzinger, J., à paraître (printemps 2017). Bauxite mining in Africa. Transnational Corporate Governance and Development. International Political Economy Series. London, Palgrave. 